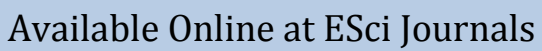 \\ International Journal of Phytopathology \\ ISSN: 2305-106X (Online), 2306-1650 (Print)
}

http://www.escijournals.net/phytopathology

\section{EFFECT OF OSMOTIC AND MATRIC POTENTIALS ON SCLEROTINIA MINOR AND SCLEROTINIA SCLEROTIORUM VIRULENCE ON PEANUT}

\author{
aAhmed Abd-Elmagid*, bRobert Hunger, bCarla Garzón, cMark Payton, dHo-Jong Ju, bHassan Melouk \\ a Department of Plant Pathology, Assiut University, Egypt. \\ b Entomology and Plant Pathology Department, Oklahoma State University, Stillwater, OK, USA. \\ c Statistics Department, Oklahoma State University, Stillwater, OK, USA. \\ d Department of Agricultural Biology, Plant Medicinal Research Center, Chonbuk National University, Republic of South Korea.
}

\section{A B S T R A C T}

The effect of osmotic and matric potentials on mycelial growth, sclerotia production, germination, and virulence of two isolates of Sclerotinia sclerotiorum, and one isolate of $S$. minor were studied on potato dextrose agar (PDA) media adjusted with $\mathrm{KCl}$, glycerol, or agar. Osmotic potentials created by $\mathrm{KCl}$ and glycerol significantly reduced vegetative growth of the three isolates. On matrically adjusted PDA, vegetative growth of the three isolates was not negatively affected by matric stress up to $-3.5 \mathrm{MPa}$. When $\mathrm{KCl}$ was the osmoticum, sclerotia number did not follow a consistent pattern. However, sclerotia number decreased when osmotic stress created by glycerol was increased. Matric stress was not a consistent factor affecting sclerotia production by both species. However, the highest levels of matric stress -3.0 and -3.5 MPa significantly reduced sclerotia production by both species. In general, there was a trend toward lower sclerotial germination with increasing osmotic and matric stress. Pathogenicity of S. minor and S. sclerotiorum on the peanut cultivar (Okrun) was reduced by high concentrations of $\mathrm{KCl}$. Mycelia of both species produced at high matric potential -3.5 MPa did not differ in pathogenicity on Okrun compared with mycelia grown on non-amended PDA. In water-stressed-Okrun, induced by polyethylene glycol 8000, the Area under Disease Progress Curve (AUDPC) was significantly decreased. The relevance of these results to the behavior of S. minor and S. sclerotiorum, and their pathogenicity on peanut is discussed.

Keywords: Water potential; Peanut; Sclerotia; Sclerotinia species.

\section{INTRODUCTION}

Sclerotinia blight of peanut (Arachis hypogaea L.) caused by the soilborne fungi Sclerotinia minor Jagger and $S$. sclerotiorum (Lib.) de Bary was first reported in the United States in Virginia in 1971 (Kokalis-Burelle et al. 1997). Sclerotinia blight has become a widespread problem in Virginia, North Carolinia, Oklahoma and Texas (Smith et al. 2006). S. minor and S. sclerotiorum survive in soil mainly by producing sclerotia on infected plants (Wu et al. 2008). Infection occurs primarily through eruptive germination of sclerotia that gives rise to white, fluffy mycelia that infect stems and pegs of peanut. Many factors affect survival and germination of sclerotia of the two species in the field (Wu et al. 2008).

* Corresponding Author:

Email: aabdelmagid77@gmail.com

(C) 2015 ESci Journals Publishing. All rights reserved.
Constant soil temperature for 3 weeks or more at $35^{\circ} \mathrm{C}$ reduces survival of sclerotia. Other factors such as sclerotial position and duration in soil, sclerotial shape, soil gases or chemicals, activities of other microorganisms and nutrition affect survival of sclerotia (Adams, 1975; Abwai and Grogan, 1975, 1979; Huang and Kozub, 1994; Imolehin et al., 1980; Burgess \& Hepworth, 1996). Temperature and moisture are significant factors affecting development of diseases caused by species of Sclerotinia spp. (Willets and Wong, 1980). Viability of sclerotia also declines rapidly over time in moist soil (i.e., low water stress) (Abwai and Grogan, 1979). Almost $100 \%$ of the sclerotia of $S$. sclerotiorum were totally decayed when soil was flooded with water for 24 to 45 days. In general, sclerotia of $S$. minor survive better in dry soil than in moist soil, and better in shallow rather than at a deeper depth in soil 
where higher moisture usually exists (Imolehin et al., 1980). Lower soil moisture (i.e., high water stress) in lettuce fields increased sclerotia survival, and sclerotia only survive short periods in saturated soils at $0 \mathrm{MPa}$ (Hao et al., 2003). Sclerotia viability decreased in soil with water potentials equal to or higher than $-0.02 \mathrm{Mpa}$ as soil temperature increased from $15^{\circ} \mathrm{C}$ to $40^{\circ} \mathrm{C}$. Sclerotia of $S$. minor can germinate directly at soil moisture levels between -0.03 and -1.5 Mpa (Imolehin et al. 1980), while sclerotia of $S$. sclerotiorum germinate between -0.6 and $0 \mathrm{Mpa}$ (Duniway et al. 1977). Optimum radial growth occurred on basal medium with osmotic potential of $-1.2 \mathrm{Mpa}$ and at $-10 \mathrm{Mpa}$ there was no growth (Imolehin et al.1980). Sclerotia of $S$. minor produced over the range of -0.1 to $-4.35 \mathrm{Mpa}$ did not differ significantly in its ability to germinate eruptively when moistened (Imolehin et al. 1980). Sclerotia of $S$. minor and S. sclerotiorum maintained within wet soil $(\geq$ $0.02 \mathrm{Mpa}$ ) for four weeks at $40^{\circ} \mathrm{C}$ did not germinate, while sclerotia maintained within dry soil $\leq-10$ Mpa for 4 weeks at $40^{\circ} \mathrm{C}$ were viable (Matheron \& Porchas, 2005). Most research on the effect of water potential on S. minor and S. sclerotiorum was performed on isolates infecting lettuce under environmental factors significantly different from those found in peanut fields. Our research was performed with Sclerotinia isolates pathogenic on peanut. Development of more effective integrated disease management strategies for control of Sclerotinia blight peanut could be improved benefit from new knowledge of the factors that affect the biology of the host, the fungi, and their interaction. Therefore, the objectives of this study were to: 1) study the effect of water potential on the vegetative growth and sclerotia production of S. minor and S. sclerotiorum, 2) determine germination success of sclerotia produced on nutrient media at various water potentials, 3) study the pathogenicity of $S$. minor and S. sclerotiorum produced on media at various matric and osmotic potentials, and 4) determine the impact of water stress on the infection rate of peanut with S. minor and S. sclerotiorum.

\section{MATERIALS AND METHODS}

Plant materials and fungal cultures: The cultivar Okrun, a sclerotinia blight-susceptible runner type peanut, was used in this study. Seeds were germinated on wet filter paper at $30^{\circ} \mathrm{C}$ in an incubator for two days, and then planted in pots (10 $\mathrm{cm}$ dia) containing a 2:1:1 (sand: soil: shredded peat moss). Plants were grown in a climate-controlled greenhouse, watered daily, and fertilized with $0.45 \%$ ammonium nitrate solution weekly, starting on the third week after planting, to promote the production of highly succulent stems. Three Sclerotinia isolates were used, including one isolate of $S$. minor from peanut and two $S$. sclerotiorum isolates, one from peanut grown in Nebraska, and the other was isolated from pumpkin fruit that was bought from a supermarket in Stillwater, OK. Isolates were maintained at $25 \pm 2{ }^{\circ} \mathrm{C}$, in darkness, on potato dextrose agar (Difco Laboratories, Detroit, MI) containing 100 ppm of streptomycin sulfate (SPDA).

Preparation of media at various water potentials: SPDA containing $100 \mathrm{ppm}$ of streptomycin sulfate was used as a basal medium. SPDA medium was osmotically modified over the range of -0.5 to $-4 \mathrm{MPa}$ with potassium chloride (Ritchie et al. 2006) or glycerol (Dallyn \& Fox 1980) and sterilized by autoclaving for 20 minutes. Total water potential was the sum of the water potential of the SPDA - $0.34 \mathrm{MPa}$ and the osmotic potential of the added osmotica (potassium chloride or glycerol) (Campbell \& Gardner, 1971; Dallyn \& Fox, 1980). Osmotic potential was calculated according to (Liddell, 1993). The actual osmotic potential of all media was checked by Vapor Pressure Osmometer (VAPRO 5520, Wescor, Utah, USA). Various matric potentials of SPDA were adjusted by granulated agar (Fisher Scientific, Fair Lawn, New Jersey). Matric potentials of media equivalent to $-1,-1.5$, $-2.0,-2.5,-3.0$, and $-3.5 \mathrm{MPa}$ at $25^{\circ} \mathrm{C}$ were determined using a Vapor Pressure Osmometer (Wescor). The total matric potential was the sum of the water potential of SPDA and the matric potential of the added agar.

Mycelial growth and sclerotia production on nutrient medium: Petri dishes containing $15 \mathrm{ml}$ of nutrient medium were each inoculated in the center with a 3-mm -dia mycelial disc taken from the periphery of 2-day-old cultures of $S$. minor and $S$. sclerotiorum grown on SPDA. Inoculated plates were incubated at $25 \pm 2{ }^{\circ} \mathrm{C}$ in darkness. Radial growth $(\mathrm{mm})$ of colony was measured up to four days after inoculation. Sclerotia were harvested from 21-days old cultures with the aid of camel hair brush. Harvested sclerotia were dried at $22^{\circ} \mathrm{C}$ for two weeks in a desiccator containing anhydrous $\mathrm{CaSO}_{4}$. Sclerotia from each $9.0-\mathrm{cm}$ plate were counted. This experiment was conducted twice with five plates as replications in each treatment.

Viability of sclerotia: Sclerotia produced under different osmotic and matric potentials were tested for viability by plating on SPDA medium. Before plating, 
sclerotia were surface sanitized with a sodium hypochlorite solution 1.0\% (Melouk et al., 1999). For each treatment, five sclerotia were plated on each of five plates, and incubated at a room temperature of $25 \pm 2{ }^{\circ} \mathrm{C}$ in darkness. Percentage of sclerotial germination was determined after 7 days of incubation.

Pathogenicity of mycelia produced on media at various water potentials: Plant inoculations were performed on six-to-eight-weeks old peanut plants (Faske et al., 2006). A total of eight pots (4 replicates) were used for each of the osmotic and matric potentials. Plants were then placed in humidity chambers $(150 \times 60 \times 60 \mathrm{~cm})$ built from PVC pipe and clear plastic. Temperature was maintained at $19 \pm 2{ }^{\circ} \mathrm{C}$ at night and $26 \pm 2{ }^{\circ} \mathrm{C}$ during the day, and relative humidity was maintained at 95 to $100 \%$. Light in the incubation chamber was adequate (13.5 $\mu \mathrm{mol} / \mathrm{m}^{2} / \mathrm{sec}$ ) to sustain healthy plants. Inoculated plants were watered as needed for the duration of the experiments. Starting three days after inoculation, lesion length on infected stems was measured and recorded, and continued every 24 hours until day 7 post inoculation. The plants were then left to dry for 1 week in the chambers to facilitate production of sclerotia on infected tissue. To facilitate further drying, the infected stems were clipped at soil level and placed in brown paper bags for one week more. Sclerotia were collected from both the stem surface and from within the pith cavity of the stem, and quantified based on number and weight. Experimental design was a random complete block design (RCBD) with 4 replicates.

The effect of water stress on the infection of peanut by $\boldsymbol{S}$. minor and $\boldsymbol{S}$. sclerotiorum: Total number of plants in the experiment was 72, representing 9 treatments and 8 replicates. Each treatment had 8 plants that were placed in a humidity chamber $(58.7 \mathrm{x}$ $42.9 \times 40 \mathrm{~cm}$ ). Six-to-eight-week old Okrun plants that received PEG 8000 solutions were prepared for inoculation as described by Faske et al. (2006). Water stress was applied to plants using polyethylene glycol 8000 (Union Carbide Chemicals and Plastics). PEG solutions of various water potentials were prepared according to (Michel and Kaufmann, 1973) (Table 1). PEG 8000 was applied by pouring each solution into the bottom of its assigned plastic chamber on the fourth week after planting. In the non-inoculated control group water was used to keep seedlings well irrigated. Six-to-eight week old plants were prepared for inoculation as described by Faske et al. (2006).

Table 1. Required concentrations of polyethylene glycol (PEG 8000) solutions to attain corresponding water stress on peanut plants at $25^{\circ} \mathrm{C}$.

\begin{tabular}{cc}
\hline$\%$ PEG 8000 & Osmotic stress in $\mathrm{MPa}$ \\
\hline 0 & $<-0.05$ \\
5 & -0.05 \\
10 & -0.15 \\
15 & -0.30 \\
20 & -0.49 \\
25 & -0.73 \\
30 & -1.03 \\
35 & -1.37 \\
40 & -1.76 \\
\hline
\end{tabular}

Statistical analysis: The experiment was performed using the same methods with each of the three isolates. Lesion length was taken at the fourth day post inoculation. This experiment was repeated once. Statistical analyses were done using SAS 9.3 (SAS Institute). Analysis of variance procedures (PROC MIXED) were conducted to determine the effects of the factors in question. Simple effects of factors were compared with planned comparisons with a SLICE option in an LSMEANS statement. Pairwise comparisons of least square means were made when overall significance was attained at a 0.05 level.

\section{RESULTS}

Mycelial growth of Sclerotinia isolates on SPDA with various water potentials: In osmotic potential $\left(\psi_{s}\right)$ experiments, mycelial growth response of Sclerotinia isolates to $\left(\psi_{s}\right)$ was similar for the two osmotica (Table 2). On both $\mathrm{KCl}$ and glycerol amended SPDA, the vegetative growth of $S$. sclerotiorum (peanut isolate) was consistently reduced at osmotic stress values below -1.5 MPa (Table 2). On both $\mathrm{KCl}$ and glycerol amended SPDA, the vegetative growth of $S$. sclerotiorum (pumpkin isolate) was consistently reduced at osmotic stress values below -2.5 MPa (Table 2). On both $\mathrm{KCl}$ and glycerol amended SPDA, the vegetative growth of $S$. minor was significantly reduced at osmotic stress values below -1.5 MPa (Table 2). This suppression of vegetative growth suggests that $S$. sclerotiorum (pumpkin isolate) may tolerate higher levels of osmotic stress and survive better than $S$. sclerotiorum (peanut isolate) and $S$. minor under similar conditions. In matric potential $\left(\psi_{\mathrm{m}}\right)$ studies, 
vegetative growth of $S$. minor, S. sclerotiorum (peanut isolate), and $S$. sclerotiorum (pumpkin isolate) was not negatively affected by matric stress of up to $-3.5 \mathrm{MPa}$ (Table 3).

Table 2. Mean area under mycelial growth progress curve (AUMGC) for $S$. sclerotiorum (peanut isolate), $S$. sclerotiorum (pumpkin isolate) and $S$. minor grown on SPDA with different osmotic potentials $\left(\psi_{\mathrm{s}}\right)$ using $\mathrm{KCl}$ and Glycerol.

\begin{tabular}{cccc}
\hline Isolates & $\begin{array}{c}\text { Osmotic } \\
\text { potentials (MPa) }\end{array}$ & $\begin{array}{c}\text { AUMGC }^{1} \\
(\mathrm{KCl})\end{array}$ & $\begin{array}{c}\text { AUMGC }^{2} \\
\text { (Glycerol) }\end{array}$ \\
\hline SS $^{3}$ & -0.34 & $19.190 \mathrm{~b}^{4}$ & $21.580 \mathrm{~b}^{5}$ \\
SS & -0.50 & $18.480 \mathrm{~b}$ & $25.010 \mathrm{a}$ \\
SS & -1.00 & $20.440 \mathrm{a}$ & $23.310 \mathrm{ab}$ \\
SS & -1.50 & $19.120 \mathrm{~b}$ & $18.630 \mathrm{c}$ \\
SS & -2.00 & $19.090 \mathrm{~b}$ & $15.495 \mathrm{~d}$ \\
SS & -2.50 & $14.925 \mathrm{c}$ & $14.826 \mathrm{~d}$ \\
SS & -3.00 & $13.980 \mathrm{c}$ & $10.560 \mathrm{ef}$ \\
SS & -3.50 & $9.830 \mathrm{~d}$ & $12.092 \mathrm{e}$ \\
\hline SS & -4.00 & $10.390 \mathrm{~d}$ & $10.160 \mathrm{f}$ \\
SSP & -0.34 & $16.910 \mathrm{c}$ & $28.560 \mathrm{a}$ \\
SSP & -0.50 & $19.040 \mathrm{~b}$ & $26.810 \mathrm{a}$ \\
SSP & -1.50 & $21.325 \mathrm{a}$ & $17.886 \mathrm{~b}$ \\
SSP & -2.00 & $22.255 \mathrm{a}$ & $13.490 \mathrm{c}$ \\
SSP & -2.50 & $18.750 \mathrm{~b}$ & $13.270 \mathrm{c}$ \\
SSP & -3.00 & $16.980 \mathrm{c}$ & $8.380 \mathrm{~d}$ \\
SSP & -3.50 & $13.500 \mathrm{~d}$ & $7.745 \mathrm{~d}$ \\
SSP & -4.00 & $12.905 \mathrm{~d}$ & $5.220 \mathrm{~d}$ \\
\hline SM & -0.34 & $16.155 \mathrm{a}$ & $29.710 \mathrm{a}$ \\
SM & -0.50 & $16.514 \mathrm{a}$ & $29.050 \mathrm{a}$ \\
SM & -1.00 & $16.670 \mathrm{a}$ & $26.240 \mathrm{~b}$ \\
SM & -1.50 & $13.015 \mathrm{~b}$ & $22.328 \mathrm{c}$ \\
SM & -2.00 & $9.945 \mathrm{c}$ & $20.102 \mathrm{~d}$ \\
SM & -2.50 & $10.545 \mathrm{c}$ & $20.090 \mathrm{~d}$ \\
SM & -3.00 & $7.795 \mathrm{~d}$ & $14.121 \mathrm{e}$ \\
SM & -3.50 & $7.895 \mathrm{~d}$ & $11.972 \mathrm{f}$ \\
SM & -4.00 & $5.425 \mathrm{e}$ & $09.630 \mathrm{~g}$ \\
\hline
\end{tabular}

${ }_{1}^{1}$ Means of area under mycelial growth progress curve values on $\mathrm{KCl}$ amended SPDA.

2 Means of area under growth progress curve values on glycerol amended SPDA.

${ }^{3}$ SS, S. sclerotiorum (peanut isolate), SSP, S. sclerotiorum (pumpkin isolate), and SM, S. minor.

4,5Means with the same letter in the same column for each isolate were not significantly different at $\mathrm{P} \geq 0.05$ level of significance.
Table 3. Mean area under mycelial growth progress curve (AUMGC) for $S$. sclerotiorum (peanut isolate), $S$. sclerotiorum (pumpkin isolate) and $S$. minor grown on SPDA with different matric potentials $\left(\psi_{\mathrm{m}}\right)$.

\begin{tabular}{ccc}
\hline Isolates & Matric potentials in MPa & AUMGC $^{1}$ \\
\hline SS $^{2}$ & -0.34 & $21.95 \mathrm{~b}^{3}$ \\
SS & -1.00 & $22.81 \mathrm{a}$ \\
SS & -1.50 & $21.91 \mathrm{~b}$ \\
SS & -2.00 & $22.24 \mathrm{ab}$ \\
SS & -2.50 & $22.88 \mathrm{a}$ \\
SS & -3.00 & $22.46 \mathrm{ab}$ \\
SS & -3.50 & $22.89 \mathrm{a}$ \\
\hline SSP & -0.34 & $21.62 \mathrm{bc}$ \\
SSP & -1.00 & $24.49 \mathrm{a}$ \\
SSP & -1.50 & $22.03 \mathrm{bc}$ \\
SSP & -2.00 & $22.75 \mathrm{ab}$ \\
SSP & -2.50 & $21.58 \mathrm{bc}$ \\
SSP & -3.00 & $21.14 \mathrm{bc}$ \\
SSP & -3.50 & $20.34 \mathrm{c}$ \\
\hline SM & -0.34 & $26.93 \mathrm{bc}$ \\
SM & -1.00 & $28.62 \mathrm{a}$ \\
SM & -1.50 & $26.85 \mathrm{bc}$ \\
SM & -2.00 & $26.25 \mathrm{~cd}$ \\
SM & -2.50 & $25.49 \mathrm{~d}$ \\
SM & -3.00 & $25.39 \mathrm{~d}$ \\
SM & -3.50 & $27.52 \mathrm{~b}$ \\
\hline
\end{tabular}

${ }^{1}$ Means of area under mycelial growth progress curve values on matrically amended SPDA.

${ }^{2} \mathrm{SS}$, S. sclerotiorum (peanut isolate), SSP, S. sclerotiorum (pumpkin isolate), and SM, S. minor

${ }^{3}$ Means with the same letter in the same column for each isolate were not significantly different at $\mathrm{P} \geq 0.05$.

Sclerotial production on nutrient media: Different levels of osmotic potentials $\left(\psi_{\mathrm{s}}\right)$ created by $\mathrm{KCl}$ and glycerol significantly $(P=0.05)$ affected sclerotia number produced by the three Sclerotinia isolates (Table 4). In general, when $\mathrm{KCl}$ was the osmoticum, sclerotia number did not follow a consistent pattern. However, when glycerol was the osmoticum, sclerotia number decreased when osmotic stress increased (Table 4). In matric potential $\left(\psi_{\mathrm{m}}\right)$ studies, different levels of $\left(\psi_{\mathrm{m}}\right)$ significantly affected the mean sclerotia number produced by the three isolates (Table 5).

Sclerotial production on nutrient media: Different levels of osmotic potentials $\left(\psi_{\mathrm{s}}\right)$ created by $\mathrm{KCl}$ and glycerol significantly $(P=0.05)$ affected sclerotia number produced by the three Sclerotinia isolates (Table 4). In general, when 
$\mathrm{KCl}$ was the osmoticum, sclerotia number did not follow a consistent pattern. However, when glycerol was the osmoticum, sclerotia number decreased when osmotic stress increased (Table 4$)$. In matric potential $\left(\psi_{\mathrm{m}}\right)$ studies, different levels of $\left(\psi_{\mathrm{m}}\right)$ significantly affected the mean sclerotia number produced by the three isolates (Table 5).

Table 4. Mean of sclerotia number of $S$. sclerotiorum (peanut isolate), S. sclerotiorum (pumkin isolate) and $S$. minor produced on SPDA amended to various osmotic potentials using $\mathrm{KCl}$ and glycerol.

\begin{tabular}{ccll}
\hline Isolates & $\begin{array}{c}\text { Osmotic } \\
\text { potentials in } \\
\text { MPa }\end{array}$ & $\begin{array}{c}\text { Sclerotia } \\
\text { number }{ }^{1} \mathrm{KCl}\end{array}$ & $\begin{array}{l}\text { Sclerotia } \\
\text { number } \\
\text { Glycerol }\end{array}$ \\
\hline SS $^{3}$ & -0.34 & $9.8 \mathrm{bcd}^{4}$ & $20.40 \mathrm{a}^{5}$ \\
SS & -0.50 & $8.6 \mathrm{~cd}$ & $19.20 \mathrm{ab}$ \\
SS & -1.00 & $9.6 \mathrm{bcd}$ & $17.80 \mathrm{abc}$ \\
SS & -1.50 & $15.2 \mathrm{a}$ & $15.40 \mathrm{bc}$ \\
SS & -2.00 & $11.2 \mathrm{bcd}$ & $15.20 \mathrm{bc}$ \\
SS & -2.50 & $10.8 \mathrm{bcd}$ & $14.00 \mathrm{~cd}$ \\
SS & -3.00 & $8.2 \mathrm{~d}$ & $13.80 \mathrm{~cd}$ \\
SS & -3.50 & $11.6 \mathrm{bc}$ & $10.00 \mathrm{ed}$ \\
SS & -4.00 & $11.8 \mathrm{~b}$ & $9.60 \mathrm{e}$ \\
\hline SSP & -0.34 & $13.8 \mathrm{de}$ & $70.80 \mathrm{a}$ \\
SSP & -0.50 & $17.0 \mathrm{cde}$ & $48.20 \mathrm{~b}$ \\
SSP & -1.00 & $20.2 \mathrm{bcd}$ & $39.60 \mathrm{bc}$ \\
SSP & -1.50 & $22.0 \mathrm{abc}$ & $36.80 \mathrm{c}$ \\
SSP & -2.00 & $28.4 \mathrm{a}$ & $35.20 \mathrm{c}$ \\
SSP & -2.50 & $22.8 \mathrm{abc}$ & $33.00 \mathrm{~cd}$ \\
SSP & -3.00 & $25.2 \mathrm{ab}$ & $36.00 \mathrm{~cd}$ \\
SSP & -3.50 & $20.4 \mathrm{bcd}$ & $25.00 \mathrm{~d}$ \\
SSP & -4.00 & $13.0 \mathrm{e}$ & $24.60 \mathrm{~d}$ \\
\hline SM & -0.34 & $460.2 \mathrm{~d}$ & $1018.80 \mathrm{a}$ \\
SM & -0.50 & $546.0 \mathrm{~d}$ & $992.20 \mathrm{a}$ \\
SM & -1.00 & $492.4 \mathrm{~d}$ & $948.00 \mathrm{a}$ \\
SM & -1.50 & $735.2 \mathrm{bc}$ & $924.40 \mathrm{a}$ \\
SM & -2.00 & $776.8 \mathrm{~b}$ & $731.20 \mathrm{~b}$ \\
SM & -2.50 & $760.8 \mathrm{~b}$ & $682.80 \mathrm{~b}$ \\
SM & -3.00 & $788.0 \mathrm{~b}$ & $644.00 \mathrm{~b}$ \\
SM & -3.50 & $1069.6 \mathrm{a}$ & $535.20 \mathrm{c}$ \\
SM & -4.00 & $593.6 \mathrm{~cd}$ & $520.00 \mathrm{c}$ \\
\hline
\end{tabular}

${ }_{1}^{1}$ Means of sclerotia number produced/plate on $\mathrm{KCl}$ amended SPDA.

${ }^{2}$ Means of sclerotia number produced/plate on glycerol amended SPDA.

${ }^{3}$ SS, S. sclerotiorum (peanut isolate), SSP, S. sclerotiorum (pumpkin isolate), and SM, S. minor

4,5Means with the same letter in the same column for each isolate were not significantly different at $P \geq 0.05$.
Table 6. Percentage of sclerotia germination of $S$. sclerotiorum (peanut isolate), S. sclerotiorum (pumpkin isolate) and S. minor, produced on SPDA with various osmotic potentials of 0.0 to $-4.0 \mathrm{MPa}$.

\begin{tabular}{ccc}
\hline Isolates & $\begin{array}{c}\text { Osmotic Potentials } \\
\text { in MPa }\end{array}$ & $\begin{array}{c}\text { \% Sclerotia } \\
\text { germination }\end{array}$ \\
\hline SS & -0.34 & $100.0 \mathrm{a}^{3}$ \\
SS & -0.50 & $100.0 \mathrm{a}$ \\
SS & -1.00 & $100.0 \mathrm{a}$ \\
SS & -1.50 & $100.0 \mathrm{a}$ \\
SS & -2.00 & $100.0 \mathrm{a}$ \\
SS & -2.50 & $95.0 \mathrm{a}$ \\
SS & -3.00 & $95.0 \mathrm{a}$ \\
SS & -3.50 & $90.0 \mathrm{a}$ \\
SS & -4.00 & $90.0 \mathrm{a}$ \\
\hline SSP & -0.34 & $100.0 \mathrm{a}$ \\
SSP & -0.50 & $100.0 \mathrm{a}$ \\
SSP & -1.00 & $100.0 \mathrm{a}$ \\
SSP & -1.50 & $100.0 \mathrm{a}$ \\
SSP & -2.00 & $100.0 \mathrm{a}$ \\
SSP & -2.50 & $100.0 \mathrm{a}$ \\
SSP & -3.00 & $95.0 \mathrm{ab}$ \\
SSP & -3.50 & $85.0 \mathrm{bc}$ \\
SSP & -4.00 & $80.0 \mathrm{c}$ \\
\hline SM & -0.34 & $100.0 \mathrm{a}$ \\
SM & -0.50 & $100.0 \mathrm{a}$ \\
SM & -1.00 & $100.0 \mathrm{a}$ \\
SM & -1.50 & $95.0 \mathrm{ab}$ \\
SM & -2.00 & $95.0 \mathrm{ab}$ \\
SM & -2.50 & $95.0 \mathrm{ab}$ \\
SM & -3.00 & $90.0 \mathrm{ab}$ \\
SM & -3.50 & $80.0 \mathrm{bb}$ \\
SM & -4.00 & $5 \mathrm{ab}$ \\
\hline & & anchded
\end{tabular}

${ }^{1}$ Percentage of sclerotia germination on SPDA amended to different osmotic potentials.

${ }^{2}$ SS, S. sclerotiorum, SSP, S. sclerotiorum (pumpkin), and SM, S. minor

${ }^{3}$ Means with the same letter in the same column for each isolate were not significantly different at $\mathrm{P} \geq 0.05$.

Sclerotial germination: Germination of sclerotia of $S$. sclerotiorum (pumpkin isolate) and $S$. minor produced at different levels of osmotic potentials $\left(\psi_{\mathrm{s}}\right)$ created by $\mathrm{KCl}$ was significantly $(P=0.05)$ affected (Table 6). However, germination of the sclerotia of $S$. sclerotiorum (peanut isolate) produced at various levels of $\left(\psi_{\mathrm{s}}\right)$ was not affected (Table 6). 
Virulence of mycelia produced on media at various water potentials: In osmotic potential $\left(\psi_{s}\right)$ studies, mycelia of $S$. sclerotiorum (peanut isolate), and S. minor produced on different $\left(\psi_{s}\right)$ were inconsistent in its virulence against the runner peanut cv. Okrun (Table 8). Only mycelia of $S$. sclerotiorum (pumpkin isolate) produced at osmotic stress at -2.0 MPa and lower were statistically less virulent (Table 8). In matric potential studies $\left(\psi_{\mathrm{m}}\right)$, mycelia of $S$. minor and $S$. sclerotiorum produced at different matric levels were inconsistent in its virulence against peanut cv. Okrun (Table 9).

Table 7. Percentage of sclerotia germination of $S$. sclerotiorum (peanut isolate), S. sclerotiorum (pumpkin isolate) and S. minor, produced on SPDA with various matric potentials of 0.0 to $-3.5 \mathrm{MPa}$.

\begin{tabular}{ccc}
\hline Isolates & $\begin{array}{c}\text { Matric potentials } \\
\text { in MPa }\end{array}$ & $\begin{array}{c}\text { \% Sclerotia } \\
\text { germination }\end{array}$ \\
\hline SS $^{2}$ & -0.34 & $100.0 \mathrm{a}^{3}$ \\
SS & -1.00 & $100.0 \mathrm{a}$ \\
SS & -1.50 & $100.0 \mathrm{a}$ \\
SS & -2.00 & $100.0 \mathrm{a}$ \\
SS & -2.50 & $100.0 \mathrm{a}$ \\
SS & -3.00 & $85.0 \mathrm{~b}$ \\
SS & -3.50 & $80.0 \mathrm{~b}$ \\
\hline SSP & -0.34 & $100.0 \mathrm{a}$ \\
SSP & -1.00 & $100.0 \mathrm{a}$ \\
SSP & -1.50 & $100.0 \mathrm{a}$ \\
SSP & -2.00 & $100.0 \mathrm{a}$ \\
SSP & -2.50 & $95.0 \mathrm{a}$ \\
SSP & -3.00 & $90.0 \mathrm{a}$ \\
SSP & -3.50 & $55.0 \mathrm{~b}$ \\
\hline SM & -0.34 & $100.0 \mathrm{a}$ \\
SM & -1.00 & $99.0 \mathrm{a}$ \\
SM & -1.50 & $99.0 \mathrm{a}$ \\
SM & -2.00 & $99.0 \mathrm{a}$ \\
SM & -2.50 & $99.0 \mathrm{a}$ \\
SM & -3.00 & $98.0 \mathrm{a}$ \\
SM & -3.50 & $98.0 \mathrm{a}$ \\
\hline
\end{tabular}

1Percentage of sclerotia germination on SPDA amended to different matric potentials.

${ }^{2} \mathrm{SS}, \quad$ S. sclerotiorum (peanut); SSP, S. sclerotiorum (pumpkin); SM, S. minor

${ }^{3}$ Means with the same letter in the same column for each isolate were not significantly different at $\mathrm{P} \geq 0.05$.

Effect of plant water stress on infection of peanut: Water stressed seedlings of cultivar Okrun, as measured by determining the relative water content (RWC) (Teulat et al. 1997), differed significantly $(P=0.05)$ in their susceptibility to infection by each of the three Sclerotinia isolates (Table 10). Stressed plants exhibited less disease when inoculated with $S$. minor or S. sclerotiorum (peanut isolate). AUDPC produced by both isolates decreased as the water stress level increased (Table 10).

Table 8. Mean area under disease progress curve (AUDPC) on "Okrun" inoculated with mycelia of $S$. sclerotiorum (peanut isolate), S. sclerotiorum (pumpkin isolate) and S. minor produced on osmotic amended SPDA.

\begin{tabular}{ccl}
\hline Isolates & $\begin{array}{c}\text { Osmotic } \\
\text { Potentials in MPa }\end{array}$ & AUDPC 1 \\
\hline SS $^{2}$ & -0.34 & $16.108 \mathrm{ab}^{3}$ \\
SS & -0.50 & $15.731 \mathrm{abc}$ \\
SS & -1.00 & $12.956 \mathrm{cbd}$ \\
SS & -1.50 & $17.781 \mathrm{a}$ \\
SS & -2.00 & $9.844 \mathrm{~d}$ \\
SS & -2.50 & $12.200 \mathrm{cbd}$ \\
SS & -3.00 & $11.000 \mathrm{~d}$ \\
SS & -3.50 & $10.419 \mathrm{~d}$ \\
\hline SSP & -0.34 & $40.375 \mathrm{ab}$ \\
SSP & -0.50 & $47.032 \mathrm{a}$ \\
SSP & -1.00 & $18.000 \mathrm{c}$ \\
SSP & -1.50 & $30.875 \mathrm{~b}$ \\
SSP & -2.00 & $18.813 \mathrm{c}$ \\
SSP & -2.50 & $19.281 \mathrm{c}$ \\
SSP & -3.00 & $14.656 \mathrm{c}$ \\
SSP & -3.50 & $15.094 \mathrm{c}$ \\
\hline SM & -0.34 & $33.500 \mathrm{a}$ \\
SM & -0.50 & $33.815 \mathrm{a}$ \\
SM & -1.00 & $30.313 \mathrm{ab}$ \\
SM & -1.50 & $30.719 \mathrm{ab}$ \\
SM & -2.00 & $31.219 \mathrm{ab}$ \\
SM & -2.50 & $24.813 \mathrm{~b}$ \\
SM & -3.00 & $26.406 \mathrm{~b}$ \\
SM & -3.50 & $29.438 \mathrm{ab}$ \\
\hline
\end{tabular}

${ }^{1}$ Means of area under disease progress curve values caused by mycelia produced on $\mathrm{KCl}$ amended SPDA.

${ }^{2}$ SS, S. sclerotiorum (peanut isolate), SSP, S. sclerotiorum (pumpkin isolate), and SM, S. minor

${ }^{3}$ Means with the same letter in the same column for each isolate were not significantly different at $\mathrm{P} \geq 0.05$.

In case of $S$. sclerotiorum (pumpkin isolate), AUDPC decreased significantly $(P=0.05)$ as the water stress applied on plants increased but there was an eruption in the amount of the disease observed on plants when water stressed to -1.76 MPa (Table 10). 
Our results have shown that stressed peanut plants exhibited less disease when inoculated with the peanut isolate of $S$. minor or S. sclerotiorum. Our search of the literature has found no previous research that examined the effect of the status of water hydration on peanut and its infection by $S$. minor and $S$. sclerotiorum. Short-term droughts for days or weeks during the growing season may predispose plants to diseases (Schoeneweiss, 1975). For example, larger cankers were induced by Lasiodiplodia theobromae on water stressed Cornus florida L. (Mullen et al. 1991), by Hypoxylon prunatum on water stressed Populus tremuloides (Bagga and Smalley, 1969), and drought stress increased the severity of Botryosphaeria blight of Pistacia vera caused by Botryosphaeria dothidea (Ma et al. 2001).

Table 9. Mean area under disease progress curve (AUDPC) on "Okrun" inoculated with mycelia of S. sclerotiorum (peanut isolate), S. sclerotiorum (pumpkin isolate) and $S$. minor produced on metrically amended SPDA.

\begin{tabular}{ccc}
\hline Isolates & $\begin{array}{c}\text { Matric Potentials in } \\
\text { MPa }\end{array}$ & AUDPC $^{1}$ \\
\hline SS & -0.34 & $19.925 \mathrm{a}^{3}$ \\
SS & -1.00 & $21.743 \mathrm{a}$ \\
SS & -1.50 & $23.893 \mathrm{a}$ \\
SS & -2.00 & $28.050 \mathrm{a}$ \\
SS & -2.50 & $20.225 \mathrm{a}$ \\
SS & -3.00 & $28.431 \mathrm{a}$ \\
SS & -3.50 & $25.225 \mathrm{a}$ \\
\hline SSP & -0.34 & $15.937 \mathrm{a}$ \\
SSP & -1.00 & $15.543 \mathrm{ab}$ \\
SSP & -1.50 & $15.718 \mathrm{ab}$ \\
SSP & -2.00 & $15.293 \mathrm{ab}$ \\
SSP & -2.50 & $13.718 \mathrm{~b}$ \\
SSP & -3.00 & $15.469 \mathrm{ab}$ \\
SSP & -3.50 & $15.398 \mathrm{ab}$ \\
\hline SM & -0.34 & $20.093 \mathrm{a}$ \\
SM & -1.00 & $16.587 \mathrm{a}$ \\
SM & -1.50 & $15.550 \mathrm{a}$ \\
SM & -2.00 & $17.293 \mathrm{a}$ \\
SM & -2.50 & $16.931 \mathrm{a}$ \\
SM & -3.00 & $16.906 \mathrm{a}$ \\
SM & -3.50 & $13.468 \mathrm{a}$ \\
\hline
\end{tabular}

${ }^{1}$ Means of area under disease progress curve values caused by mycelia produced on metrically amended SPDA using agar.

${ }^{2} \mathrm{SS}$, S. sclerotiorum (peanut isolate), SSP, S. sclerotiorum (pumpkin isolate), and SM, S. minor.

${ }^{3}$ Means with the same letter in the same column for each isolate were not significantly different at $\mathrm{P} \geq 0.05$.
Table 10. Mean area under disease progress curve (AUDPC) on Okrun cultivar under water stress and infected by $S$. sclerotiorum (peanut isolate), $S$. sclerotiorum (pumpkin isolate) and S. minor

\begin{tabular}{ccc}
\hline Isolates & Water stress in MPa & AUDPC $^{1}$ \\
\hline SS $^{2}$ & 0.00 & $35.406 \mathrm{a}^{3}$ \\
SS & -0.05 & $35.953 \mathrm{a}$ \\
SS & -0.15 & $32.891 \mathrm{ab}$ \\
SS & -0.30 & $26.563 \mathrm{abc}$ \\
SS & -0.49 & $26.734 \mathrm{abc}$ \\
SS & -0.73 & $24.844 \mathrm{bc}$ \\
SS & -1.03 & $22.078 \mathrm{c}$ \\
SS & -1.37 & $21.031 \mathrm{c}$ \\
SS & -1.76 & $21.016 \mathrm{c}$ \\
\hline SSP & 0.00 & $34.109 \mathrm{a}$ \\
SSP & -0.05 & $32.344 \mathrm{a}$ \\
SSP & -0.15 & $33.047 \mathrm{a}$ \\
SSP & -0.30 & $30.588 \mathrm{ab}$ \\
SSP & -0.49 & $30.438 \mathrm{ab}$ \\
SSP & -0.73 & $27.766 \mathrm{bc}$ \\
SSP & -1.03 & $25.844 \mathrm{~cd}$ \\
SSP & -1.37 & $23.828 \mathrm{~d}$ \\
SSP & -1.76 & $38.625 \mathrm{e}$ \\
\hline SM & 0.00 & $33.463 \mathrm{a}$ \\
SM & -0.05 & $30.225 \mathrm{ab}$ \\
SM & -0.15 & $29.038 \mathrm{ab}$ \\
SM & -0.30 & $24.797 \mathrm{bc}$ \\
SM & -0.49 & $19.984 \mathrm{~cd}$ \\
SM & -0.73 & $19.234 \mathrm{~cd}$ \\
SM & -1.03 & $13.531 \mathrm{~d}$ \\
SM & -1.37 & $14.703 \mathrm{~d}$ \\
SM & -1.76 &
\end{tabular}

${ }_{1}^{1}$ Means of area under disease progress curve values on Okrun under water stress created by PEG8000.

${ }^{2} \mathrm{SS}$, S. sclerotiorum (peanut isolate), SSP, S. sclerotiorum (pumpkin isolate), and SM, S. minor

${ }^{3}$ Means with the same letter in the same column for each isolate were not significantly different at $\mathrm{P} \geq 0.05$.

Our results contradict these observations. Our data indicate that water stressed plants had smaller lesions compared with non-water stressed plants or plants that were under less water stress. This information can be used in disease management by applying less irrigation to infected peanut plants or on plants grown in infested soils. Reduction in mycelial growth of $S$. minor and $S$. sclerotiorum under increased osmotic stress suggests that the reduced growth of both species may partly explain the reduction in AUDPC on plants under high 
level of water stress. Sclerotinia sclerotiorum (pumpkin isolate), caused larger lesions when the water stress level increased. There was no published data, to our knowledge, concerning effects of water potential on mycelial growth, sclerotial number and germination of Sclerotinia sclerotiorum and Sclerotinia minor collected from peanut fields. Therefore, this study is the first to show the negative effects of osmotic and matric stress on mycelial growth and sclerotial formation of the two Sclerotinia species. Furthermore, this study stated for the first time the effect of water stress on the infection of peanut by $S$. sclerotiorum and S. minor.

Our results contradict these observations. Our data indicate that water stressed plants had smaller lesions compared with non-water stressed plants or plants that were under less water stress. This information can be used in disease management by applying less irrigation to infected peanut plants or on plants grown in infested soils. Reduction in mycelial growth of $S$. minor and $S$. sclerotiorum under increased osmotic stress suggests that the reduced growth of both species may partly explain the reduction in AUDPC on plants under high level of water stress. Sclerotinia sclerotiorum (pumpkin isolate), caused larger lesions when the water stress level increased. There was no published data, to our knowledge, concerning effects of water potential on mycelial growth, sclerotial number and germination of Sclerotinia sclerotiorum and Sclerotinia minor collected from peanut fields. Therefore, this study is the first to show the negative effects of osmotic and matric stress on mycelial growth and sclerotial formation of the two Sclerotinia species. Furthermore, this study stated for the first time the effect of water stress on the infection of peanut by $S$. sclerotiorum and S. minor.

\section{DISCUSSION}

Sudden changes in the external conditions can disrupt the homeostasis and normal physiology of all living organisms. Therefore, cells have developed complex systems to identify the adverse changes of their environment, and rapidly generate defense mechanism to survive environmental stresses (Lushchak, 2011). The pattern of mycelial growth of $S$. minor and $S$. sclerotiorum, presented in the results section, was similar to that observed by Ferrin and Stanghellini (2006) with Monosprascus cannonballus, which indicates that the observed responses were caused by changes in osmotic stress rather than by toxicity of the osmotica. Also, the mycelial growth responses of $S$. minor and $S$. sclerotiorum to different osmotic stress in this study are similar to those previously observed for other soil borne pathogens (Ritchie et al. 2006). For instance, mycelial growth of Rhizoctonia solani (Kumar et al. 1999), Gaeumannomyces graminis (Grose et al. 1984), Typhula idanoensis and Typhula incarnata (Bruehl and Cunfer 1971), Macrophomina phaseolina (Cervantes-Garcia et al. 2003), and Aspergillus niger and Fusarium moniliforme (Subbarao et al. 1993) was reduced when osmotic stress increased.

Solutes present in agar medium trap water molecules, therefore water will not be available to $\mathrm{S}$. minor and S. sclerotiorum. The energy spent by the fungi to obtain water molecules from the medium is increased as the solute concentrations in the agar medium increase, and therefore reduction of fungal growth occurs. Ionic solutes such as $\mathrm{KCl}$ and $\mathrm{NaCl}$ and non-ionic solutes such glycerol and sucrose have been used in several water potential studies involving various plant pathogenic fungi like F. solani (Palacios et al, 2014), Phytophthora cryptogea and Fusarium moniliforme (Woods and Duniway, 1986), Verticillium dahlia (Ioannou et al. 1977) and the biocontrol agent Coniothyrium minitans (Jones et al, 2011). In this research, S. minor and S. sclerotiorum isolates grew on $\mathrm{KCl}$ and glycerol adjusted PDA over all levels of the osmotica tested (Table 2). The ability of a fungus to grow under osmotic stress and the exact optimal water potential depends on the fungal species and in some cases on the osmoticum, temperature, or other factors in the environment (Cook, 1981). Mycelial growth under $\mathrm{KCl}$ osmotic stress may result from uptake of potassium ions and its accumulation by microbial cells, which lower the water potential of the protoplasm to a value more ideal for cellular processes, or may increase turgor and hence acceleration of growth (Olaya et al. 1996). On matrically modified SPDA, S. sclerotiorum (peanut isolate) had the highest mycelial growth at -3.5 $\mathrm{MPa}$, however, there were no significant differences over the range -2.0 to $-3.5 \mathrm{MPa}$. S. sclerotiorum (pumpkin isolate) and S. minor grew best at -1.0 MPa. However, area under mycelial growth curve (AUMGC) values produced by the three isolates at the lowest matric potential were greater than those recorded at the lowest osmotic potential used in this study. Moreover, the mycelial growth of the three isolates have not been inhibited at the lowest matric potential used in this study, $-3.5 \mathrm{MPa}$, which is lower than the permanent wilting point of mesophytic higher plants -1.5 MPa; (Slayter, 1967). 
In general, matric stress has not shown to be a consistent factor affecting the number of sclerotia produced by the three Sclerotinia isolates. However, there appears to be a statistical trend to support that the highest level of matric stress, $-3.5 \mathrm{MPa}$, favorably affected the number of sclerotia produced by $S$. sclerotiorum and $S$. minor (Table 5). Total sclerotia production by any test isolate was greater on glycerol amended PDA than on $\mathrm{KCl}$ amended PDA (Table 4). This may be due to the utilization of the glycerol as a carbon source by $S$. sclerotiorum and S. minor (Sommers et al. 1970). On matrically amended SPDA, the three isolates of $S$. sclerotiorum and $S$. minor produced the biggest numbers of sclerotia on -3 and -3.5 Mpa (Table 5). This indicates these isolates of $S$. sclerotiorum and $S$. minor were well adapted to wider ranges of soil water potentials well beyond the limits of their peanut host, provided that other environmental factors are conducive. Also, osmotic stress forces the isolates of $S$. sclerotiorum and $S$. minor to produce sclerotia as survival structure. This could be one of the factors involved in its fitness as a soil-borne plant pathogen (Ritchie et al. 2006).

The difference in sclerotial germination between the two isolates of $S$. sclerotiorum in response to osmotic stress $(\psi s)$ suggests that within each species there may exist ecotypes with variability in their response to environmental factors. This needs future research. Evaluating matric potential $(\psi \mathrm{m})$, significant differences were observed between treatments for $S$. sclerotiorum isolates but not for $S$. minor. At the lowest $(\psi \mathrm{m})-3.5$ $\mathrm{Mpa}$, the percentage of sclerotia germination was $80 \%$ for $S$. sclerotiorum (peanut isolates), $55 \%$ for $S$. sclerotiorum (pumpkin isolate) and $98 \%$ for $S$. minor. Ability of sclerotia to germinate at low $(\psi s)$ is perhaps due to solute uptake by the sclerotium causing a reduction in its internal osmotic potential, and so allowing maintenance of germination processes (Cook and Al-Hamdani, 1986). In this study, sclerotial formation and germination of $S$. minor and $S$. sclerotiorum occurred at $(\psi \mathrm{s})$ and $(\psi \mathrm{m})$ lower than those at which most crops seed germination and root development are curtailed (-1.4 to $-2.0 \mathrm{MPa})$; (Tommerup, 1984). This could be of importance to understand the ecological factors that could affect pathogenicity as well as saprophytic behavior.

Different matric potentials did not significantly affect AUDPCs produced by the three isolates (Table 9). No research has been done before to investigate the effect of osmotic and matric potentials on the virulence of $S$. minor and S. sclerotiorum. Few studies in the literature investigated the effect of water potential on the virulence of plant pathogenic fungi. Cervantes-Garcia et al. (2003) observed a reduction in the pathogenicity of Macrophomina phaseolina on seeds of common beans, as $\mathrm{NaCl}$ concentrations increased in potato-glucose-agar medium. The results reported herein show that $S$. minor and $S$. sclerotiorum can grow vegetatively under relatively low water potentials. The ability of $S$. minor and S. sclerotiorum to grow in a wide range of water potentials indicates the presence of adaptive mechanisms for life under variable environmental conditions. Adapting to a wide range of water potentials may be a strategy to survive as a saprophyte.

Our data have shown that stressed peanut plants exhibited less disease when inoculated with the peanut isolate of $S$. minor or S. sclerotiorum. Our search of the literature has found no previous research that examined the effect of the status of water hydration on peanut and its infection by $S$. minor and S. sclerotiorum. However, Schoeneweiss (1975) has found that short-term droughts for days or weeks during the growing season may predispose plants to diseases. Other examples, larger cankers were induced by Lasiodiplodia theobromae on water stressed Cornus florida L. (Mullen et al. 1991), by Hypoxylon prunatum on water stressed Populus tremuloides (Bagga and Smalley 1969), and drought stress increased the severity of Botryosphaeria blight of Pistacia vera caused by Botryosphaeria dothidea (Ma et al. 2001). Our results contradict these observations. Our data indicate that water stressed plants had smaller lesions compared with non-water stressed plants grown under near optimal conditions of peanut growth. This information can be used in disease management by applying less irrigation to infected peanut plants or on plants grown in infested soils. Reduction in mycelial growth of $S$. minor and $S$. sclerotiorum under increased osmotic stress suggests that the reduced growth of both species may partly explain the reduction in AUDPC on plants under high level of water stress.

\section{ACKNOWLEDGMENT}

This research was funded by the department of Entomology and Plant Pathology, Oklahoma State University, and the Oklahoma Agricultural Experiment Station, Stillwater, Oklahoma 74078. 


\section{REFRENCES}

Abwai, G.S., and R.G. Grogan. 1975. Source of primary inoculum and effects of temperature and moisture on infection of beans by Whetzelinia sclerotiorum. Phytopathol. 65: 300-309.

Abwai, G.S., and R.G. Grogan. 1979. Epidemiology of diseases caused by Sclerotinia species. Phytopathol. 69: 899-904.

Adam, P.B. 1975. Factors affecting survival of Sclerotinia sclerotiorum in soil. Plant Dis. Rep. 59: 599-603.

Bagga, D.K. and E.B. Smalley. 1969. Factors affecting canker development on Populus tremuloides artificially inoculated with Hypoxylon pruinatum. Can. J. of. Bot. 47: 907-914.

Brownell, K.H. and R.W. Schneider. 1985. Roles of matric and osmotic components of water potential and their interaction with temperature in the growth of Fusarium oxysporum in synthetic media and soil. Phytopathol. 75: 53-57.

Bruehl, G.W. and B. Cunfer, 1971. Physiologic and environmental factors that affect the severity of snow mold of wheat. Phytopathology 61: 792-799.

Burgess, D.R. and G. Hepworth. 1996. Biocontrol of Sclerotinia stem rot (Sclerotinia minor) in sunflower by seed treatment with Gliocladium virens. Plant Pathol. 45: 583-592.

Campbell, G.S., and W.H. Gardner, 1971. Psychrometric measurement of soil water potential: temperature and bulk density effects. Soil Science Society of America, Proceedings. 35: 8-12.

Cervantes-Garcia, D. and J. S. Padilla-Ramirez and J. Simpson and N. Mayek-Perez, 2003. Osmotic potential effects on in vitro growth, morphology and pathogenicity of Macrophomina phaseolina. J. of Phytopathol.151: 456-462.

Cook, R.C. and A.M. Al-Hamadani, 1986. Water relations of sclerotia and other infective structures. Pages 49-63 in: Water, Fungi and Plants. P. G. Ayres and L. Boddy, eds. Cambridge University Press, Cambridge.

Cook, R.J. 1981. Water relations in the biology of Fusarium. Pages 236-244 in: Fusarium: Diseases, Biology, and Taxonomy. P. E. Nelson, T. A. Toussoun, and R. J. Cook, eds. The Pennsylvania Satate University Press, University Park.

Dallyn, H. and A. Fox. 1980. Spoilage of material of reduced water activity by xerophilic fungi. In: Gould, G. H., Corry, E. L., eds. Microbial growth and survival in extreme environments. London and New York: Academic Press. 129-139pp.

Duniway, J.M. and G.S. Abawi and J.R. Steadman, 1977. Influence of soil moisture on the production of apothecia by Whetzelinia sclerotiorum. (Abstr). Proceedings of the Amer. Phytopathol. Soci. 4:115.

Faske, T. and H.A. Melouk and M.E. Payton. 2006. Comparison of Sclerotinia minor inocula for differentiating the reaction of peanut genotypes to Sclerotinia blight. Peanut Sci. 33: 7-11.

Ferrin, D.M. and M.E. Stanghellini. 2006. Effect of water potential on mycelial growth and perithecial production of Monosprascus cannonballus in vitro. Plant Pathol. 55: 421-426.

Griffin, D.M. 1981. Water and microbial stress. In: Alexander A, ed. Advances in microbial ecology 5. New York. Plenum Publishing Co. 91-136pp.

Grose, M.J. and C.A. Parker and K. Sivasithamparam, 1984. Growth of Gaeumannomyces graminis var. Tritici in soil: effects of temperature and water potential. Soil Biol. Biochem. 16: 211-216.

Hao, J.J. and K.V. Subbarao and J.M. Duniway. 2003. Germination of Sclerotinia minor and $S$. sclerotiorum sclerotia under various soil moisture and temperature combinations. Phytopathol. 93: 443-450.

Huang, H.C. and G.C. Kozub. 1994. Longevity of normal and abnormal sclerotia of Sclerotinia sclerotiorum. Plant Dis. 78: 1164-166.

Imolehin, E.D. and R. G. Grogan and J. M. Duniway. 1980. Effect of temperature and moisture tension on growth, sclerotial production, germination, and infection by Sclerotinia minor. Phytopathol. 70: 1153-1157.

Ioannou, N. and R.W. Scheider and R.G. Grogan and J.M. Duniway, 1977. Effect of water potential and temperature on growth, sporulation, and production of microsclerotia by Verticillium dahlia. Phytopathol. 67: 637-644.

Jennings, D.H. 1995. The physiology of fungal nutrition. Cambridge, UK: Cambridge University Press. PP. 622.

Jennings, D.H. and R.M. Burke. 1990. Compatible solutesthe mycological dimension and their role as physiological buffering agents. New Phytol. 116: 277-283.

Jones, E. E. and A. Stewart and J. M. Whipps. 2011. Water potential effects Coniothyrium minitans gowth, 
germination and parasitism of Sclerotinia sclerotiorum sclerotia. Fungal Biol. 115 (9): 871-81.

Kokalis-Burelle, N. and D.M. Porter and R. RodríguezKabana and D.H. Smith and and P. Subrahmanyam. 1997. Sclerotinia Blight. Pages 34-36 in: Compendium of Peanut Diseases. Second edition. American Phytopathol. Soci. St. Paul, Minnesota.

Kumar, S. and K. Sivasithamparam and J.S. Gill and M.W. Sweetingham. 1999. Temperature and water potential effects on growth and pathogenocoty of Rhizoctonia solani Ag-11 to lupine. Can. J. Microbiol. 45: 389-395.

Lang, A.R.G., 1967. Osmotic coefficients and water potentials of sodium chloride solutions from 0 to $40^{\circ} \mathrm{C}$. Aus. J. of Chem. 20: 2017-2023.

Liddell, C.M. 1993. Measurement and control of soil temperature and water potential. In: Singleton, $\mathrm{L}$. L., Mihail, J. D., Rush, C. M, eds. Methods for research on soilborne phytopathogenic fungi. Eds,. The American Phytopathol. soci..

Lushchak, V. I. 2011. Adaptive response to oxidative stress: bacteria, fungi, plants and animals. Comp. Biochem. Physiol. C Toxicol. Pharmacol. 153: 175-190.

Ma, Z. and D.P. Morgan and T.J. Michailides, 2001. Effect of water stress on Botryosphaeria blight of pistachio caused by Botryosphaeria dothidea. Plant Dis. 85: 745-749.

Magan, N. and J. M. Lynch. 1986. Water potential, growth and cellulolysis of fungi involved in decomposition of cereal residues. J. Gen. Microbiol. 132: 1181-1187.

Matheron, M.E. and M. Porchas. 2005. Influence of soil temperature and moisture on eruptive germination and viability of Sclerotinia minor and S. sclerotiorum. Plant Dis. 89: 50-54.

Melouk, H.A. and C. Bown and S.S. Aboshosha. 1999. A procedure for isolation and determination of incidence of Sclerotinia minor in peanut seed. Alexanderia J. Agric. Res. 44: 261-270.

Michel, B.E. and M.R. Kaufmann. 1973. The osmotic potential of polyethylen glycol 6000. Plant Physiol. 51: 914-916.

Mullen, J.M. and C.H. Gilliam and A.K. Hagan and G. Morgan-Jones, 1991. Canker of dogwood caused by Lasiodiplodia theobromae, a disease influenced by drought stress or cultivar selection. Plant Dis. 75: 886-889.

Olaya, G. and G.S. Abawi. 1996. Effect of water potential on mycelial growth and on production and germination of sclerotia of Macrophomina phaseolina. Plant Dis. 80: 1347-1350.

Palacios, S. and F. Casasnovas and M. L. Ramirez and M. M. Reynoso and A.M. Torres. 2014. Impact of water potential on growth and germination of Fusarium solani soilborne pathogen of peanut. Brazil. J. Microbiol. 45 (3): 1105-1112.

Ramirez, M.L. and S.N. Chulze and N. Magan. 2004. Impact of osmotic and matric water stress on germination, growth, mycelial water potentials and endogenous accumulation of sugars and sugar alcholos in Fusarium graminearum. Mycologia. 96 (3): 470-478.

Ritchie, F. and M.P. McQuilken and R.A. Bain. 2006. Effect of water potential on mycelial growth, sclerotial production, and germination of Rhizoctonia solani from potato. Mycol. Res. 110: 725-733.

Schoeneweiss, D. F. 1975. Predisposition, stress, and plant diseases. Annual Review of Phytopathology 13: 193-211.

Slayter, R. D. 1967. Plant-water relationships. Academic Press, New York.

Smith, D. L. and J. E. Hollowell and T. G. Isleib and B. B. Shew, 2006. Analysis of factors that influence the epidemiology of sclerotinia minor on peanut. Plant Dis. 90: 1425-1432.

Sommers, L. E. and R. F. Harris and F. N. Dalton and W. R. Gardner, 1970. Water potential relations of three root-infecting Phytophthora species. Phytopathol. 60: 932-934.

Subbarao, K. V. and T. J. Michailides and D. P. Morgan, 1993. Effects of osmotic potential and temperature on growth of two pathogens of figs and a biocontrol agent. Phytopathol. 83: 14541459.

Teulat, B. and P. Monneveux and J. Wery and B.I. Souyris and A. Charrier and D. This, 1997. Relatioships between relative water content and growth parameters under stress in barely: a QTL study. New Phytol. 137: 99-107.

Tommerup, I. C. 1984. Effect of soil water potential on spore germination by vesiculararbuscular mycorrhizal fungi. Trans. British Mycol. Soci. 83: 193-202.

Willets, H. J. and J. A. L. Wong. 1980. The biology of Sclerotinia sclerotiorum, S. trifoliorum, S. minor with emphasis on specific nomenclature. The Bot. Rev. 46: 101-165. 
Int. J. Phytopathol. 04 (03) 2015. 147-158

Woods, D.M. and J.M. Duniway. 1986. Some of effects of water potential on growth, turgor, and repiration of Phytophthora cryptogea and Fusarium moniliforme. Phytopathol. 76: 1248-1253.
Wu, B. M. and K. V. Subbarao and Y. B. Liu. 2008. Comparative survival of sclerotia of Sclerotinia minor and S. sclerotiorum. Phytpathol. 98: 659665. 